\title{
Stimulated Mobilization of Monocyte Mac-1 and p150,95 Adhesion Proteins from an Intracellular Vesicular Compartment to the Cell Surface
}

Linda J. Miller, Dorothy F. Bainton, ${ }^{*}$ Niels Borregaard, ${ }^{\ddagger}$ and Timothy A. Springer

Laboratory of Membrane Immunochemistry, Dana-Farber Cancer Institute and Department of Pathology, Harvard Medical School, Boston, Massachusetts 02115; *Department of Pathology, University of California Medical Center, San Francisco, California 94143; ${ }^{\ddagger}$ Department of Hematology C, Gentofte Hospital, Copenhagen, Denmark

\begin{abstract}
Monocytes were stimulated to increase their cell surface quantity of leukocyte adhesion proteins p150,95 and Mac-1 by the chemoattractant formyl-methionyl-leucyl-phenylalanine, or other mediators such as platelet-derived growth factor, tumor necrosis factor, C5a, and leukotriene B4. Dose-response curves indicated variations in the sensitivity of monocytes and granulocytes to these mediators. These increases were independent of protein synthesis and half-maximal at 2 min. Human alveolar and murine peritoneal macrophages, cells that had previously diapedised, could not be induced to upregulate Mac-1 or p150,95. Detergent permeabilization studies in monocytes indicated that these proteins were stored in internal latent pools, which were reduced upon stimulation. Electron microscopy utilizing rabbit antiserum against p150,95 revealed these proteins on the plasma membrane, and in intracellular vesicles and peroxidase negative granules. Together with other functional studies, these findings suggest that the mobilization of Mac-1 and p150,95 from an intracellular compartment to the plasma membrane regulates the monocytes's ability to adhere and diapedese.
\end{abstract}

\section{Introduction}

In the inflammatory response blood monocytes and granulocytes bind to endothelial cells at the inflamed site, then migrate between endothelial cells and through the basement membrane into tissue. A key question is how the first step in this process, adhesion of the monocyte (or granulocyte) to the endothelial cell, is regulated. Changes in adhesiveness of the endothelial cell, as well as in the adhesiveness of the monocyte (or granulocyte) may both contribute to this process $(1-4)$. Granulocyte accumulation in tissues kinetically precedes that of monocytes (1). This has led to the idea that different mechanisms may regulate granulocyte and monocyte infiltration. However, granulocyte infiltration is not a prerequisite for monocyte infiltration, because monocyte infiltration is normal in neutropenic patients (1).

Recently, an inherited immunodeficiency disease has been identified in which both granulocyte and monocyte diapedesis is defective (reviewed in 4,5). A family of three leukocyte adhesion glycoproteins, Mac-1, p150,95, and LFA-1 are absent from

Address reprint requests to Dr. Springer, Dana Farber Cancer Institute, 44 Binney St., (JFB-425), Boston, MA 02115.

Received for publication 20 August 1986 and in revised form 17 March 1987.

J. Clin. Invest.

(c) The American Society for Clinical Investigation, Inc.

0021-9738/87/08/0535/10 \$2.00

Volume 80, August 1987, 535-544 patient monocytes and granulocytes. These three glycoproteins are alpha/beta complexes, which utilize a common beta subunit. In deficient patients, both granulocytes and monocytes are present in the circulation at elevated or normal levels, respectively, but fail to accumulate at sterile inflammatory sites (Rebuck skin windows) and at sites of infection. Normal leukocytes transfused into patients, however, localize normally. Wound healing in deficient patients is delayed, apparently reflecting the lack of monocyte infiltration, since neutropenic patients show normal wound healing. In vitro, granulocytes and monocytes from deficient patients show decreased adherence to endothelial cells (6-9). Monoclonal antibodies (MAb) to the Mac-1 alpha subunit and the common beta subunit inhibit adherence by healthy monocytes and granulocytes to a variety of substrates including endothelial cells $(5,10-13)$. These findings suggest that monocyte and granulocyte diapedesis is regulated by a similar mechanism, in which the Mac-1, p150,95, and LFA-1 family of adhesion glycoproteins is of key importance.

In granulocytes or specifically, neutrophils, inflammatory stimuli such as formyl-methionyl-leucyl-phenylalanine ( $f$ MLP), ${ }^{1}$ C5a, and leukotriene B4 (LTB4) mobilize intracellular stores of Mac-1 and p150,95 to the cell surface (14-16). Increased surface expression of Mac-1 (and perhaps p150,95) appears to mediate granulocyte hyperadherence to endothelial cells and other substrates, and granulocyte aggregation. The intracellular storage site for Mac-1 in neutrophils has been reported to be the secondary granule (16). Monocytes, like granulocytes, have receptors for the inflammatory mediators $f$ MLP, C5a, LTB4, and platelet derived growth factor (PDGF) and in response to them chemotax, show increased adherence to endothelial cells, aggregation, and superoxide anion generation (17-20).

In monocytes the mechanism(s) that regulates mediatorstimulated increases in adhesiveness has been unclear, and whether the mechanism is similar to that in granulocytes is controversial. There have been conflicting reports that inflammatory stimuli do not increase surface expression of Mac-1 (Mo1) on monocytes (21), in keeping with their lack of secondary granules, or alternatively, that Mac-1 is increased by twofold (22), a considerably smaller increase than on granulocytes.

Here, we show that $f$ MLP induces a rapid increase in monocyte cell surface expression of Mac-1 and p150,95. Preparative procedures can also lead to temperature-dependent increases in Mac-1 and p150,95. By minimizing cell handling, we demonstrate large (4- to 12-fold) $f$ MLP-dependent increases. The $f$ MLP-stimulated increase does not require protein synthesis. The mediators PDGF, tumor necrosis factor (TNF), LTB4, and

1. Abbreviations used in this paper: $\mathrm{ACD}$, citrate phosphate dextrose adenine; FITC, fluorescein isothiocyanate; $f$ MLP, formyl-methionylleucyl-phenylalanine; LTB4, leukotriene B4; MAb, monoclonal antibody; PDGF, platelet-derived growth factor; TNF, tumor necrosis factor. 
C5a also induce increased Mac-1 expression. Permeabilization of monocytes with detergents and immunoelectron microscopy demonstrates an intracellular vesicular compartment of Mac-1 and p150,95. We propose that mobilization of this intracellular pool of Mac-1 and p150,95 to the cell surface regulates monocyte adherence to endothelial cells, contributing to monocyte localization at inflammatory sites.

\section{Methods}

Cells. Venous blood was drawn into syringes containing $0.14 \mathrm{ml} / \mathrm{ml}$ blood of (ACD; $2 \mathrm{~g}$ glucose monohydrate, $1.66 \mathrm{~g}$ sodium citrate- $5 \mathrm{H}_{2} \mathrm{O}$, $206 \mathrm{mg}$ citric acid- $\mathrm{H}_{2} \mathrm{O}, 140 \mathrm{mg} \mathrm{NaH} \mathrm{PO}_{4}$, and $17.3 \mathrm{mg}$ adenine/63 ml) and in some experiments the syringes also contained $0.1 \mathrm{ml} / \mathrm{ml}$ blood of $0.1 \mathrm{M}$ sodium EDTA, pH 7.2. 6\% dextran T-500 (Pharmacia Fine Chemicals, Piscataway, NJ) in PBS was added to syringes giving a final dextran concentration of $1 \%$. After $\sim 30 \mathrm{~min}$ at room temperature, leukocyte-rich plasma was drawn off. Cells were either stimulated immediately or prepared further as described in Results. Hypotonic lysis of erythrocytes, where indicated, was by suspension of cell pellets in icecold $\mathrm{H}_{2} \mathrm{O}$ for $25 \mathrm{~s}$, followed by addition of $1 / 9$ vol of $10 \times$ phosphatebuffered saline (PBS). Ficoll-Hypaque solutions were diluted from $16.7 \%$ Hypaque-M (Winthrop Laboratories, New York), 12.5\% Ficoll 400 (Pharmacia Fine Chemicals) with $\mathrm{H}_{2} \mathrm{O}$ to desired density. In some experiments $1 \mathrm{mM}$ EDTA pH 7.2 was added to Ficoll-Hypaque. Leukocyterich plasma (10-25 ml) was chilled on ice in a 50-ml tube, underlayed with $12 \mathrm{ml} d=1.08$ Ficoll-Hypaque, $12 \mathrm{ml} d=1.106$ Ficoll-Hypaque, and centrifuged at $1,200 \mathrm{~g}$ for $25 \mathrm{~min}$ in a centrifuge equilibrated at $4^{\circ} \mathrm{C}$ or $-5^{\circ} \mathrm{C}$. Mononuclear cells and platelets sedimented on the $d=1.08$ layer and neutrophils sedimented on the $d=1.106$ layer. Eosinophils and erythrocytes collected in the pellet. In some cases the $d=1.106$ layer was omitted. When neutrophils were stimulated with $f$ MLP, their density changed and they sedimented on the $d=1.08$ layer, in agreement with a recent report (23). Cells were diluted in at least 2 vol of Hanks'/ $10 \mathrm{mM}$ Hepes $/ 5 \mathrm{mM}$ EDTA and washed twice at $400 \mathrm{~g}$ for $7 \mathrm{~min}$. Platelets were collected from the mononuclear cell supernatant by centrifugation at $1,000 \mathrm{~g}$ for $10 \mathrm{~min}$. Normal human alveolar macrophages were obtained by bronchoalveolar lavage of healthy subjects (nonsmokers) and generously provided by Dr. R. Rose, New England Deaconess Hospital, Boston, MA. Thioglycollate elicited mouse macrophages were collected by washing the peritoneal cavity with $10 \mathrm{ml}$ of Hanks'/10 mM Hepes/10 U/ml heparin $4 \mathrm{~d}$ after injection of $1.5 \mathrm{ml}$ of Brewer's thioglycollate broth (Difco Laboratories, Detroit, MI).

Stimulants. $f$ MLP was prepared as a $10^{-3} \mathrm{M}$ stock solution in dimethylsulfoxide and diluted to 100 times the final concentration in Hanks'/10 mM Hepes. A human recombinant TNF stock solution of 1 $\mu \mathrm{g} / \mathrm{ml}$ was provided by Dr. C. Dinarello, Tufts University School of Medicine, Boston, MA. $4 \mu \mathrm{M}$ C5a (purified from activated human serum by sequential P60 gel filtration, SP Sephadex C25, and CM Sephadex chromatography) and $2 \times 10^{-6} \mathrm{M} \mathrm{LTB} 4$, were generously supplied by Dr. P. Henson, National Jewish Hospital and Research Center, Denver, CO. A platelet extract (24) with PDGF activity of $3,000 \mathrm{U} / \mathrm{ml}$ was kindly provided by Dr. C. Stiles, Dana-Farber Cancer Institute, Boston, MA. Cells were stimulated at $10^{7} / \mathrm{ml}$ if purified, or if in leukocyte-rich plasma without any further adjustment. Cells were stimulated either in the absence or presence of EDTA as indicated; upregulation of Mac-1 was usually equivalent but was sometimes greater (up to twofold) in the presence of $\mathrm{Ca}^{+2}$ and $\mathrm{Mg}^{+2}$.

Monoclonal antibodies (MAb). MAb were used as hybridoma culture supernatants, or if indicated, were used as diluted ascites fluid or were purified. Mac-1 alpha chain MAb was LM2/1 (25). p150,95 MAb SHCL3 (26) (available as LeuM5, Becton-Dickinson, Mountain View, CA) was generously provided by Dr. R. Schwarting. LFA-1 alpha chain MAb was TS1/22 (27). CR1 MAb was $20 \mu \mathrm{g} / \mathrm{ml}$ 44D (28), the generous gift of Dr. V. Nussenzweig. Mo2 MAb (1:500 ascites) was MOP9 (29). Fc gamma receptor Fc $\gamma$ R of neutrophil MAb (1:200 ascites) was CLBFcRgran 1 (30). The Mo2 and Fc gamma R MAb were M67 and M57, respectively, of the myeloid panel of the Second International Workshop on Leukocyte Differentiation (31) and were characterized therein (32). HLA-A,B MAb was W6/32 (33). HLA-DR MAb was TS1/16 (34). The platelet gplb MAb (1:500 ascites) AN51 (35) was kindly provided by Dr. A. McMichael, Oxford. Anti-mouse Mac-1 MAb was M1/70 (36). Anti-mouse H-2 MAb was $\mathrm{M} 1 / 42$ (37).

Immunofluorescence flow cytometry. Cells at $5 \times 10^{7} / \mathrm{ml}(50 \mu \mathrm{l})$ were mixed with $50 \mu \mathrm{MAb}$ for $30 \mathrm{~min}$ at $4^{\circ} \mathrm{C}$, washed thrice, suspended in $50 \mu \mathrm{l}$ FITC anti-IgG for $30 \mathrm{~min}$ at $4^{\circ} \mathrm{C}$, washed thrice, filtered through $37 \mu \mathrm{m}$ nylon monofilament mesh (Small Parts, Inc., Miami, FL), and subjected to immunofluorescence flow cytometry in an Epics $V$ as previously described (38). All washes and dilutions were in Hanks'/10 mM Hepes $/ 5 \mathrm{mM}$ EDTA $/ 10 \%$ fetal calf serum (FCS) at $4^{\circ} \mathrm{C}$. Centrifugation in microtiter plates was at $200 \mathrm{~g}$ for $5 \mathrm{~min}$. Monocyte, granulocyte, and lymphocyte populations were separated by bit map gating on forward angle and $90^{\circ}$ light scatter. Stimulation caused a decrease in monocyte and granulocyte forward angle and to a lesser extent $90^{\circ}$ light scatter; gates were adjusted accordingly. The identity of granulocyte and monocyte populations was verified in all experimental groups by staining with neutrophil Fc $\gamma \mathrm{R}$ and Mo2 MAb. Scatter-gated populations were usually pure but up to $10 \%$ contamination was considered acceptable. Fluorescence data was collected on a 3 decade log scale. The center of each fluorescent peak was determined by visual inspection and reported as channel number. Log values were converted to linear fluorescence with calibration curves obtained by analyzing fluorescent microspheres (Coulter Electronics, Hialeah, FL) with both $\log$ and linear amplification. Specific linear fluorescence was obtained by subtracting control fluorescence in which X63 IgG1 myeloma was substituted for the first MAb.

Electron microscopy. Normal human leukocytes sedimented in dextran were washed in Hanks' solution. The cells were fixed in $0.25 \%$ glutaraldehyde in $0.1 \mathrm{M}$ phosphate buffer ( $\mathrm{pH} \mathrm{7.4)}$ ) for $1 \mathrm{~h}$ at $4^{\circ} \mathrm{C}$ and were washed well in the same buffer containing $10 \%$ (wt/vol) sucrose. They were then reacted for peroxidase (39). The cells were infiltrated for 30 min with 2.1 M sucrose, embedded in the sucrose solution, frozen, and stored in liquid nitrogen. Sections were cut on a Reichert ultracut $E$. The frozen thin section techniques have been previously described (40). The primary antibody was a rabbit antiserum against immunoaffinity purified p150,95 (40a), absorbed with an equal volume of $\sim 2 \times 10^{8}$ homogenized leukocytes from a patient severely deficient in Mac-1, LFA1 , and p150,95. It was used at a 1:20 dilution and immunogold probe, goat-anti-rabbit-gold (GAR-5), (Janssen Pharmaceutica, Beerse, Belgium), was used at a 1:50 dilution. Nonimmune purified rabbit IgG was used as the control.

\section{Results}

Preliminary studies on centrifugation-induced, temperature-dependent increases in Mac-1 and p150,95. In preliminary experiments, we used mononuclear cells purified by dextran sedimentation at room temperature and Ficoll-Hypaque gradient centrifugation at $4^{\circ} \mathrm{C}$. Cells were incubated with $10^{-8} \mathrm{M} f \mathrm{MLP}$ at $37^{\circ} \mathrm{C}$, at $37^{\circ} \mathrm{C}$ alone, or held at $4^{\circ} \mathrm{C}$. After treatment, mononuclear cells were chilled to $4^{\circ} \mathrm{C}$ and labeled with MAb, fluorescein isothiocyanate (FITC)-labeled anti-mouse IgG, and subjected to quantitative immunofluorescence flow cytometry. Monocytes were resolved from lymphocytes and large granular lymphocytes by gating on forward angle and $90^{\circ}$ light scatter. In 5 of 5 different experiments, incubation with $f \mathrm{MLP}$ at $37^{\circ} \mathrm{C}$ compared to incubation at $4^{\circ} \mathrm{C}$ resulted in a four- to eightfold $(\bar{x}=5.9)$ increase in monocyte cell surface Mac-1, a four- to ninefold $(\bar{x}=6.6)$ increase in surface $p 150,95$, no increase in LFA-1, and a 1.2 to 1.5 -fold increase in the monocyte marker Mo2. However, most of the increase in cell surface Mac-1 and p150,95 was due to incubation at $37^{\circ} \mathrm{C}$ alone (the temperaturedependent increase was three- to eightfold, $\bar{x}=5.0$ for Mac-1, 
three- to eightfold, $\bar{x}=5.5$ for p150,95) and much less of the increase (1.0- to 1.6-fold) was due to the presence of $f$ MLP. Similar temperature-dependent increases in cell surface Mac-1 and p150,95 were found on granulocytes (data not shown). Some of these preliminary experiments have previously been reported $(15,32)$. Previous studies on the $\mathrm{C} 3 \mathrm{~b}$ receptor $(41)$ have shown that cell preparation, which included centrifugation at $4^{\circ} \mathrm{C}$, primed granulocytes and monocytes, but not $B$ lymphocytes, to increase $\mathrm{C} 3 \mathrm{~b}$ receptor expression upon warming to $37^{\circ} \mathrm{C}$. We found that temperature-dependent increases in Mac- 1 and p150,95 on Ficoll-Hypaque gradient purified, washed (e.g., centrifuged) monocytes were similar whether or not EDTA at a final concentration of $10 \mathrm{mM}$ was present in the blood collection medium and contact with glass was avoided, to inhibit binding of platelets to monocytes.

fMLP-dependent induction of monocyte cell surface Mac-1 and p150,95. To avoid the artifact of centrifugation or handlinginduced increases in surface antigens during incubation at $37^{\circ} \mathrm{C}$, we adopted the following protocol. Blood was collected in ACDEDTA and erythrocytes were dextran-sedimented at room temperature. The leukocyte-rich plasma was removed, divided into aliquots, and used directly. Aliquots were either held at $4^{\circ} \mathrm{C}$, at $37^{\circ} \mathrm{C}$, or at $37^{\circ} \mathrm{C}$ with $10^{-8} \mathrm{M} f \mathrm{MLP}$ for $1 / 2 \mathrm{~h}$. The aliquots were then diluted with at least 5 vol of ice-cold Hanks'/Hepes/EDTA, washed thrice, and labeled with MAb and FITC-anti-IgG. Results with unstimulated and stimulated cells are shown on the left and right sides, respectively, of Fig. 1. Monocytes, granulocytes, and lymphocytes were resolved during fluorescence flow cytometry by gating on forward angle and $90^{\circ}$ light scatter (Fig. $1 \mathrm{~A}$, $B)$. Granulocytes were identified with MAb to the neutrophil Fc $\gamma \mathrm{R}$ (CD16), and monocytes with MAb to the Mo2 antigen (CDw14) $(31,42,43)$. The accuracy of scatter gating was confirmed by the $\mathrm{Mo}^{+}, \mathrm{HLA}_{-} \mathrm{DR}^{+}, \mathrm{Fc} \mathrm{R}^{-}$phenotype of the monocyte population (Fig. $1 E, F$ ) and the Mo2-, HLA-DR ${ }^{-}$, $\mathrm{Fc}_{\gamma} \mathrm{R}^{+}$phenotype of the granulocyte population (Fig. 1I,J). With this protocol, there was no difference whether cells were incubated at $4^{\circ} \mathrm{C}$ or $37^{\circ} \mathrm{C}$ (Table I). Strikingly, inclusion of $f$ MLP in the $37^{\circ} \mathrm{C}$ incubation resulted in a four- to fivefold increase in monocyte surface Mac-1 and p150,95. The data in Table I are averaged from three different experiments, representative histograms from which demonstrate monocyte Mac-1 and p150,95 upregulation (Fig. 1 $C, D$ ). Another member of the same glycoprotein family, LFA-1, showed a slight increase (Table I, Fig. $1 C, D$ ). Three other monocyte surface proteins, Mo2, HLA-A,B, and HLA-DR, were unaffected. The CR1 on monocytes was increased by $f$ MLP stimulation, extending previous observations on cell handling and temperature-dependent upregulation on monocytes and granulocytes, and $f$ MLP-stimulated upregulation on granulocytes (41). Similar results were obtained on cells that were treated with or without $f$ MLP during a $1 / 2-h$ period of sedimentation at $37^{\circ} \mathrm{C}$ in dextran (not shown). Similar results were also obtained whether or not $10 \mathrm{mM}$ EDTA was added to the leukocyte-rich plasma.

Parallel experiments on granulocytes demonstrated large $f$ MLP-stimulated increases in cell surface Mac-1, p150,95, and CR1, with no or little increase in LFA-1, HLA-A,B and Fc gamma R (Table I, Fig. $1 G-J$ ).

Staining with a platelet-specific MAb to glycoprotein (gp)Ib was used to assess platelet binding to monocytes and granulocytes. Platelets bound to a subpopulation of unstimulated and stimulated monocytes (Fig. $1 E, F$ ) and unstimulated granulocytes (Fig. 1I). Fluorescence measurements on platelets showed
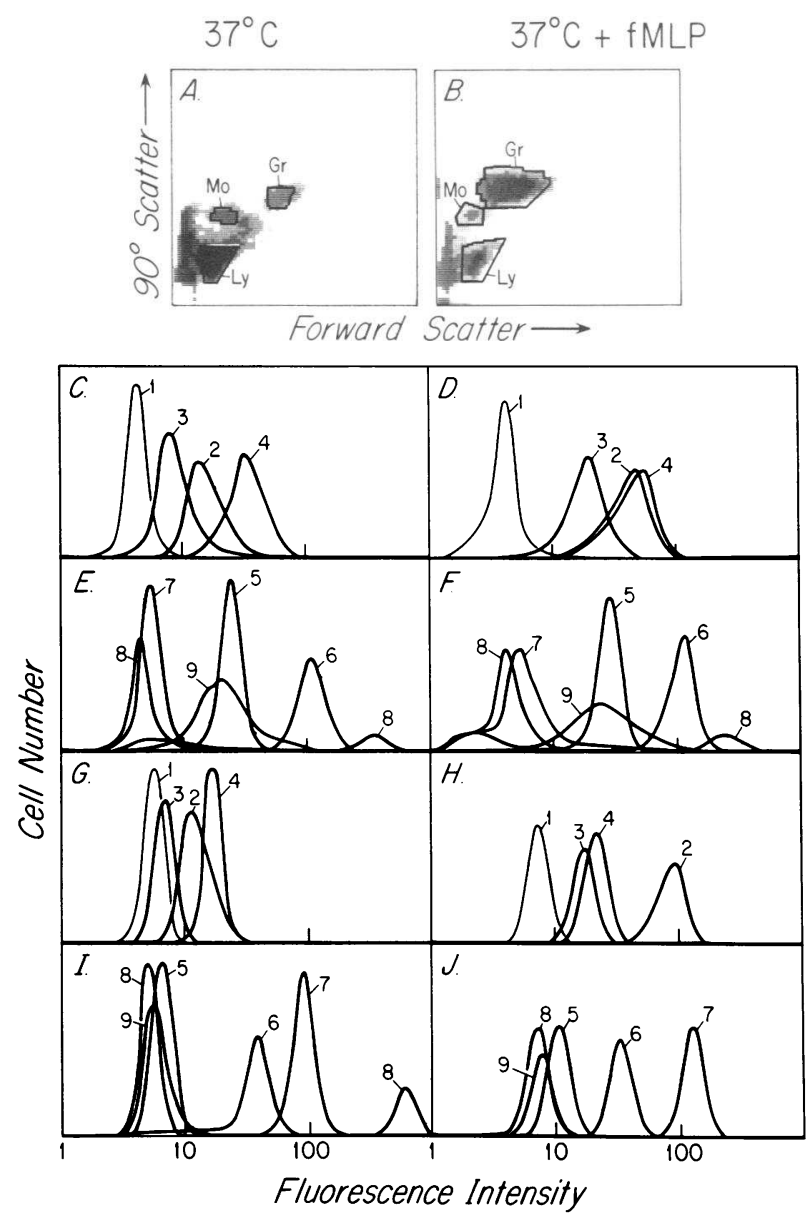

Figure 1. Effect of $f$ MLP on surface antigen expression of monocytes and granulocytes. Aliquots of leukocyte-rich plasma from dextran-sedimented blood collected in ACD/10 mM EDTA were incubated at $37^{\circ} \mathrm{C}$ with or without $10^{-8} \mathrm{M} f \mathrm{MLP}$ as indicated. Cells were then chilled, labeled with MAb and FITC anti-mouse Ig, and subjected to immunofluorescent flow cytometry. Left-hand panels represent unstimulated cells $(A, C, E, G, I)$ and right-hand panels represent $f$ MLP. stimulated cells $(B, D, F, H, J)$. Monocyte $(m o)$, granulocyte $(g r)$, and lymphocyte $(l y)$ populations were resolved by forward angle and $90^{\circ}$ light scatter; representative scatter distributions are shown in $A$ and $B$. Bit map-gated fluorescence histograms for unstimulated monocytes $(C, E), f$ MLP-stimulated monocytes $(D, F)$, unstimulated granulocytes $(G, I)$, and $f$ MLP-stimulated granulocytes $(H, J)$ were then collected. Curves in $C-J$ represent cells labeled with MAb specific for the following surface antigens: 1 , control; 2, Mac-1; 3, p150,95; 4, LFA-1; 5, Mo2; 6, HLA-A,B; 7, neutrophil Fc gamma R; 8, platelet gpIb; 9 , HLA-DR.

they are negative for Mac-1, p150,95, and LFA-1 (Table I), and immunogold staining of thin sections of platelets also showed they lack any cell surface or intracellular content of these antigens (Bainton, D., L. Miller, K. Kishimoto, and T. Springer, unpublished data). Thus, binding of platelets cannot contribute to the increased Mac-1 and p150,95 expression seen on $f$ MLP-stimulated monocytes and granulocytes.

The large increase in Mac-1 and p150,95 expression within 30 min appeared too rapidly to be accounted for by de novo protein synthesis. To test this, leukocytes were incubated with the protein synthesis inhibitor cycloheximide, then treated with $f$ MLP (Table I). The $f$ MLP-stimulated increase in monocyte and granulocyte Mac-1 and p150,95 was not inhibited by cy- 
Table I. Stimulation by fMLP of Monocyte and Granulocyte Surface Antigen Expression*

\begin{tabular}{|c|c|c|c|c|c|}
\hline \multicolumn{6}{|l|}{ Treatment } \\
\hline & $4^{\circ} \mathrm{C}$ & $37^{\circ} \mathrm{C}$ & $37^{\circ} \mathrm{C}+f \mathrm{MLP}$ & $\begin{array}{l}37^{\circ} \mathrm{C}+f \text { MLP } \\
+ \text { Cycloheximide }\end{array}$ & $\begin{array}{l}\text { Upregulation } \\
\left(37^{\circ} \mathrm{C}+f \mathrm{MLP} / 37^{\circ} \mathrm{C}\right)\end{array}$ \\
\hline & \multicolumn{4}{|c|}{ Specific fluorescence intensity } & fold \\
\hline \multicolumn{6}{|l|}{ Monocytes } \\
\hline Mac-1 & 13.1 & 9.4 & 40.2 & 47.2 & $\times 4.3$ \\
\hline $\mathrm{p} 150,95$ & 3.4 & 3.6 & 15.0 & 18.3 & $\times 4.2$ \\
\hline LFA-1 & 24.9 & 31.0 & 50.5 & 62.9 & $\times 1.6$ \\
\hline CR1 & 2.2 & 2.2 & 6.5 & 8.0 & $\times 3.0$ \\
\hline Mo2 & 15.1 & 18.1 & 21.8 & 19.2 & $\times 1.2$ \\
\hline HLA-A,B & 113.3 & 126.6 & 127 & 119.2 & $\times 1.0$ \\
\hline FcR & ND & 1.1 & 1.4 & 1.5 & - \\
\hline HLA-DR & ND & 11.1 & 12.6 & 11.4 & $\times 1.1$ \\
\hline \multicolumn{6}{|l|}{ Granulocytes } \\
\hline Mac-1 & 5.0 & 5.0 & 70.2 & 61.1 & $\times 14.0$ \\
\hline p150,95 & 1.8 & 1.2 & 9.5 & 8.9 & $\times 7.9$ \\
\hline LFA-1 & 8.9 & 9.8 & 13.2 & 12.3 & $\times 1.3$ \\
\hline CR1 & 2.3 & 2.3 & 11.9 & 10.3 & $\times 5.2$ \\
\hline Mo2 & 0.8 & 0.7 & 2.4 & 1.0 & - \\
\hline HLA-A,B & 33.5 & 34.6 & 30.4 & 30.8 & $\times 0.9$ \\
\hline FcR & 114 & 114 & 185 & 217 & $\times 1.6$ \\
\hline HLA-DR & ND & 0.0 & 0.5 & -0.8 & - \\
\hline \multicolumn{6}{|l|}{ Platelets } \\
\hline Mac-1 & 0.0 & & & & \\
\hline p150,95 & 0.0 & & & & \\
\hline LFA-1 & 0.0 & & & & \\
\hline Mo2 & 0.0 & & & & \\
\hline gplb & 15.7 & & & & \\
\hline
\end{tabular}

* Values are averages of $3 / 3$ experiments $\left(37^{\circ} \mathrm{C}, 37^{\circ} \mathrm{C}+f \mathrm{MLP}\right)$ or of $1 / 1$ experiment $\left(4^{\circ} \mathrm{C}, 37^{\circ} \mathrm{C}+f \mathrm{MLP}+\right.$ cycloheximide). Subtracted control fluorescence was 3.8 to 5.8 for monocytes, 4.4 to 8.2 for granulocytes, and 0.0 for platelets.

cloheximide, showing it must be due to preformed pools of these molecules.

The kinetics and dose response of Mac-1 and p150,95 upregulation were determined. Comparison of monocytes and granulocytes showed granulocytes were stimulated by lower concentrations of $f$ MLP than monocytes for 2/2 donors (Fig. 2 A). $f$ MLP-stimulated monocyte upregulation of Mac-1 and p150,95 was near maximal at 10 min and showed similar kinetics to granulocyte upregulation (Fig. 2 B).

$f$ MLP is an analogue of bacterial N-terminal peptides. We examined whether the results with $f$ MLP were generalizable to other inflammatory mediators. In preliminary studies, we found that defibrination of blood resulted in four- to sixfold increased Mac-1 expression on monocytes and granulocytes compared to blood collected in ACD. Defibrination activates platelets and the clotting cascade. Addition of $10 \%$ serum to leukocyte-rich plasma similarly resulted in a threefold increase in monocyte Mac-1 and p150,95, while addition of platelet-poor plasma had no effect (1.0- to 1.1-fold change). This suggested PDGF released from activated platelets was the active principle. Serum stimulated similar increases in granulocyte Mac-1 and p150,95.

The dose-response curves for four different inflammatory mediators, each on two to four different donors, is shown in Fig. 3. PDGF induced Mac-1 upregulation on both monocytes and granulocytes (Fig. $3 E, F$ ), confirming it is the factor in serum that stimulates upregulation. C5a and LTB4 stimulated modest upregulation of monocyte Mac-1 of 1.7 to 2.9-fold. In contrast to other mediators tested, C5a and LTB4 had weaker effects on monocytes than on granulocytes. TNF potently stimulated marked monocyte Mac-1 upregulation (Fig. 3 G); higher concentrations were required to stimulate granulocyte Mac-1 upregulation (Fig. $3 \mathrm{H}$ ). In these studies, we found differences between donors in the extent of monocyte Mac-1 upregulation; differences were more marked for monocytes than for granulocytes and were consistently found in independent experiments. Donor-dependent differences of severalfold in the extent of monocyte Mac-1 upregulation were also found with $f$ MLP (not shown).

We hypothesized that the rapid increase in surface Mac-1 and p150,95 on stimulated monocytes was due to mobilization to the cell surface of an intracellular pool. Translocation to the cell surface might be due to the fusion of intracellular vesicles with the plasma membrane. We predicted that permeabilization with detergents of the plasma membrane and intracellular membrane vesicles should allow antibodies to access the intracellular pool. Because permeabilization partially degraded resolution of monocytes from granulocytes by light scatter gating, mononuclear cells were first separated from granulocytes by centrifugation on Ficoll-Hypaque gradients. Cells were then held at $4^{\circ} \mathrm{C}$ or incubated at $37^{\circ} \mathrm{C}$ with $10^{-8} \mathrm{M} f$ MLP for $30 \mathrm{~min}$, 
A

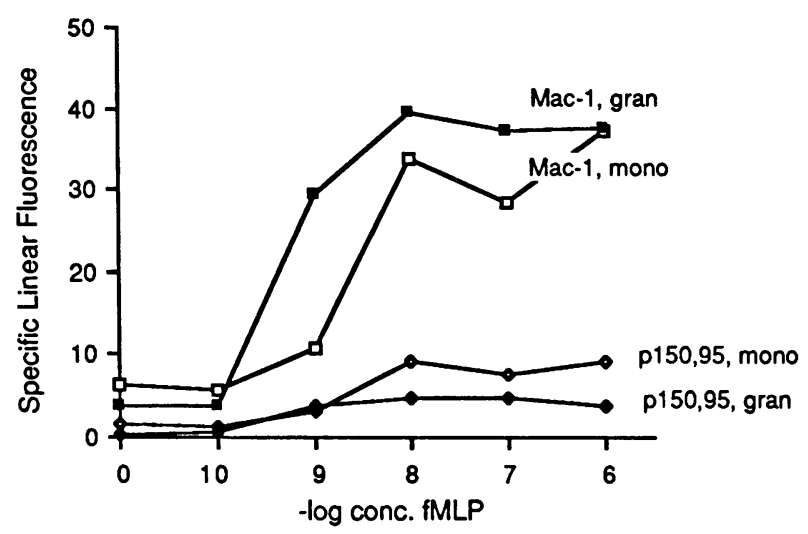

B

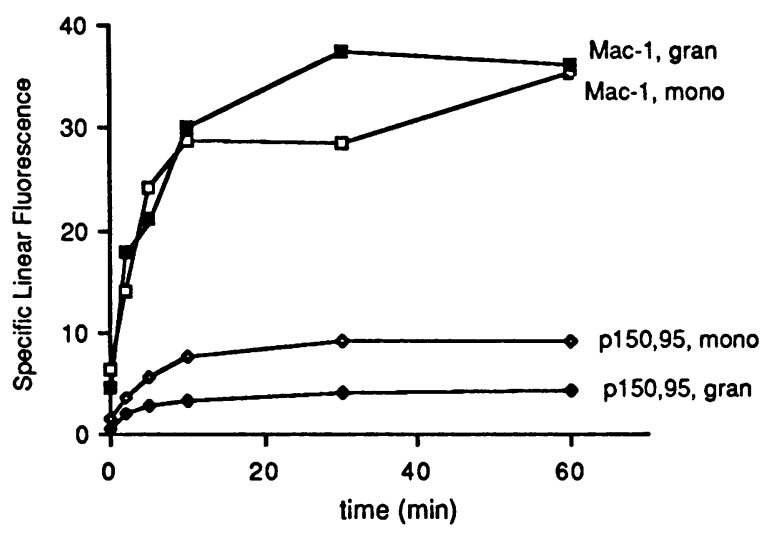

Figure 2. Dose response and kinetics of Mac-1 and p150,95 upregulation on $f$ MLP-stimulated leukocytes. Leukocyte-rich plasma from dextran-sedimented whole blood was incubated at $37^{\circ} \mathrm{C}$ with various concentrations of $f$ MLP for $30 \mathrm{~min}(A)$ or with $10^{-7} \mathrm{M} f$ MLP for various lengths of times $(B)$. Cells were washed, subjected to hypotonic lysis to remove erythrocytes, and stained for immunofluorescence flow cytometry with LM2/1 anti-Mac-1 alpha subunit MAb ( $\square$ and $\square$ ), and SHCL3 anti-p150,95 MAb ( $\diamond$ and $\diamond)$. Results shown are averages of two independent experiments with different donors. Monocytes and granulocytes were resolved by bit map gating on forward angle and $90^{\circ}$ light scatter. fixed in paraformaldehyde/glutaraldehyde followed by $\mathrm{NaBH}_{4}$ reduction, and permeabilized with Triton $\mathrm{X}-100$. MAb binding was quantitated by immunofluorescence flow cytometry.

To assess permeabilization of intracellular compartments, we measured release from neutrophils of the primary granule marker beta glucuronidase and the secondary granule marker vitamin $B_{12}$ binding protein. The release of granule contents into the supernatant and increased accessibility of Mac-1 and p150,95 to MAbs suggested permeabilization was maximal at 0.03-0.1\% Triton X-100 (Fig. 4). Immunoprecipitation of Mac1 from supernatants of permeabilized cells suggested that $0.1 \%$ Triton X-100 released only small amounts (5-15\%) of Mac-1 (data not shown).

The effect of stimulation with $f$ MLP and permeabilization with $0.1 \%$ Triton $\mathrm{X}-100$ on antibody-accessible antigen was then examined for both monocytes and granulocytes (Table II). $f$ MLP stimulated a large increase in monocyte cell surface Mac-1 and p150,95 (13.9 and 9.5-fold, respectively). When cells not incubated with $f$ MLP were permeabilized with Triton X-100, a large latent pool of Mac-1 and p150,95 became accessible, as shown by 12.5 - and 8.8 -fold increases in labeling with Mac-1 and p150,95 MAb. The quantity of Mac-1 and p150,95 accessible in unstimulated, permeabilized monocytes was similar to the quantity on the surface of stimulated, nonpermeabilized cells. Permeabilization of cells that had previously been stimulated with $f$ MLP resulted in little or no increase in accessible Mac-1 and p150,95, suggesting that the $f$ MLP-stimulated increase in surface expression of Mac-1 and p150,95 is due to surface mobilization of the latent, detergent accessible pool.

Parallel studies on granulocytes demonstrated a similar detergent accessible pool (Table II). However, more Mac-1 could be accessed after Triton X-100 permeabilization than was mobilized by $f$ MLP. This effect was consistently seen in other experiments, suggesting that mobilization by $f$ MLP of the intracellular pools of these antigens in granulocytes was incomplete.

For comparison, LFA-1 and HLA-A,B were examined. On monocytes, the amount of LFA-1 accessible to MAb showed a modest increase (twofold) after detergent permeabilization or stimulation with $f$ MLP. LFA-1 was not increased on granulocytes. There was little or no latent pool of HLA-A,B in monocytes and granulocytes.

To verify the intracellular location of Mac-1 and p150,95 in monocytes, they were subjected to immunogold staining and electron microscopy. An antiserum against immunoaffinity purified $\mathrm{p} 150,95$ protein was raised in rabbits. To remove any reac-

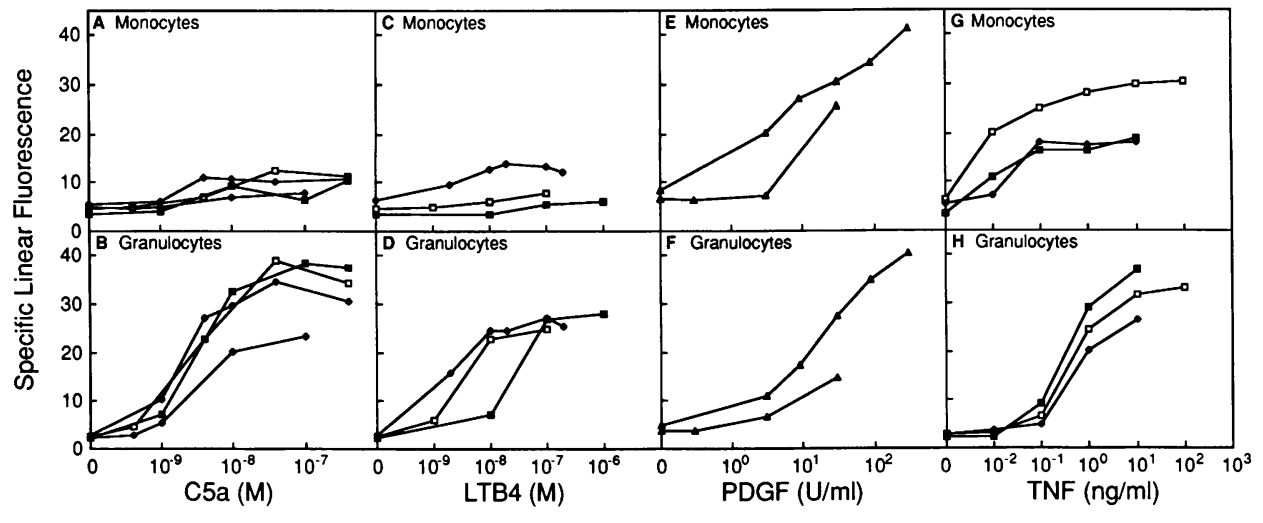

Figure 3. Concentration-dependence of mediator-stimulated Mac-1 upregulation on monocytes and granulocytes. Aliquots of leukocyte rich plasma were incubated with various concentrations of C5a, LTB4, PDGF, and TNF for $30 \mathrm{~min}$ at $37^{\circ} \mathrm{C}$. Cells were washed, subjected to hypotonic lysis to remove erythrocytes, and stained for immunofluorescence flow cytometry with $\mathrm{LM} 2 / 1$ anti-Mac-1 alpha subunit MAb. A total of six different donors were used in these experiments; each is represented with a unique symbol. 


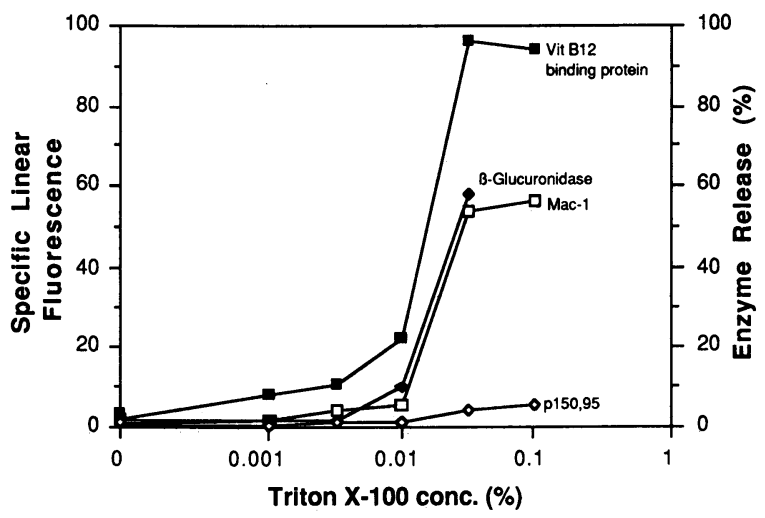

Figure 4. Effect of detergent on antigen accessibility and granule enzyme release. Isolated granulocytes were fixed as described in Results, and incubated with the indicated concentrations of Triton X-100 in Hanks'/Hepes. The cells and supernates were then separated by centrifugation, and assayed for the presence of primary $(\bullet$, beta glucuronidase) or secondary ( $n$, vitamin $B_{12}$ binding protein) granule enzymes as previously described (52). An aliquot of cells was also stained for immunofluorescent flow cytometry ( $\square$, Mac-1, and $\diamond, p 150,95$ ). The concentration of beta-glucuronidase at $0.1 \%$ Triton $\mathrm{X}-100$ was not tested.

tivity with cytoplasmic antigens contaminating the p150,95 preparation, the serum was absorbed with homogenized leukocytes from a patient severely deficient in Mac-1, LFA-1, and p150,95. The antiserum contained antibodies to both the p150,95 alpha subunit and the common beta subunit shared

Table II. Latent Pools of Mac-1 and p150,95 Are Accessible after Permeabilization of Monocytes and Granulocytes with Detergent*

\begin{tabular}{|c|c|c|c|c|c|c|}
\hline & \multirow[b]{2}{*}{$f \mathrm{MLP}$} & \multirow[b]{2}{*}{ TX-100 } & \multicolumn{4}{|c|}{ Specific fluorescence intensity } \\
\hline & & & Mac-1 & p150,95 & LFA-1 & HLA-A,B \\
\hline \multirow[t]{4}{*}{ Monocytes } & - & - & 1.6 & 1.6 & 9.8 & 46.8 \\
\hline & + & - & 22.2 & 15.3 & 21.9 & 56.5 \\
\hline & - & + & 20.0 & 14.0 & 22.5 & 59.2 \\
\hline & + & + & 23.5 & 19.3 & 26.8 & 69.4 \\
\hline \multirow[t]{4}{*}{ Granulocytes } & - & - & 2.8 & 0.6 & 3.3 & 11.4 \\
\hline & + & - & 23.4 & 3.8 & 3.1 & 7.2 \\
\hline & - & + & 30.4 & 4.0 & 3.4 & 10.8 \\
\hline & + & + & 25.5 & 3.9 & 2.6 & 6.8 \\
\hline
\end{tabular}

* Cells were prepared and treated with $f$ MLP as described in the text. Cells were fixed with an equal volume of $4 \%$ paraformaldehyde/ $0.2 \%$ or $0.5 \%$ glutaraldehyde/0.1 M sodium phosphate $\mathrm{pH} 7.3$ for $15 \mathrm{~min}$ at $4^{\circ} \mathrm{C}$, washed twice, suspended in $\mathrm{PBS} / 0.1 \%$ freshly added $\mathrm{NaBH}_{4}$ for 10 min at $20^{\circ} \mathrm{C}$, and centrifuged. Fixation and $\mathrm{NaBH}_{4}$ had no effect on antigenicity. Cells were resuspended in PBS/0.1\% Triton X-100 for 15 to $20 \mathrm{~min}$ at $20^{\circ} \mathrm{C}$ or $30 \mathrm{~min}$ at $0^{\circ} \mathrm{C}$, washed with $10 \% \mathrm{FCS} /$ Hanks'-Hepes/5 mM EDTA, labeled with MAb and FITC anti-Ig, and subjected to immunofluorescence flow cytometry. Data are averages of 3/3 experiments (Mac-1 and HLA-A,B) or 2/2 experiments (p150,95 and LFA-1). Control fluorescence (subtracted) was 3.4 to 3.8 for nonpermeabilized monocytes, 6.0 to 6.3 for permeabilized monocytes, 3.8 to 4.2 for nonpermeabilized granulocytes, and 5.1 to 5.4 for permeabilized granulocytes. with Mac-1 and LFA-1 (40a) and therefore detected Mac-1, LFA1 , and p150,95. Nichols and Bainton (39) had previously demonstrated that human blood monocytes contain two distinct populations of granules, peroxidase-positive and peroxidasenegative, in approximately equal numbers. Fig. 5 shows the localization of this family of proteins on a frozen thin section of a normal human blood monocyte that had initially been reacted for peroxidase. Label was present in peroxidase-negative vacuoles, some of which may be granules, and on the plasma membrane. Smaller quantities were found on perinuclear cisternae, and the Golgi cisternae (not illustrated) occasionally contained label. The dense peroxidase-positive granules did not contain immunogold label. Negligible gold labeling was found when the primary antiserum was replaced by normal rabbit serum.

The above studies were done on cells from blood. Since the latent pool of Mac-1 and p150,95 might be mobilized and hence exhausted during extravasation, we examined tissue macrophages. Human alveolar macrophages were obtained by bronchoalveolar lavage from a healthy subject. Mouse thioglycollateelicited peritoneal macrophages were obtained through peritoneal lavage. Cell surface antigens were quantitated with murine $M A b$ to human Mac-1, p150,95, LFA-1, and HLA-A,B and rat MAb to mouse Mac-1 and H-2K,D (Table III). Human alveolar macrophages had more p150,95 than Mac-1, consistent with previous reports that the relative quantities of these two antigens are reversed during differentiation of monocytes to tissue macrophages $(26,44)$. Incubation with $10^{-7} \mathrm{fMLP}$ resulted in no change in surface Mac-1 or p150,95 on both human and murine macrophages (Table III). Tissue macrophages have previously been shown to have $f$ MLP receptors and to chemotax to $f$ MLP (17), but in case they were not functionally active in stimulating Mac1 upregulation, we used PMA, which bypasses surface receptors by acting directly on protein kinase C. PMA has previously been shown to be a potent stimulant of increased granulocyte cell surface Mac-1 (45). PMA stimulated only a very slight (1.2-fold) increase in murine macrophage surface Mac-1 and no increase in human alveolar macrophage Mac-1 or p150,95 (Table III). Similar to thioglycollate broth-elicited peritoneal macrophages, resident murine peritoneal macrophages were found not to increase surface Mac-1 after stimulation with $f$ MLP or phorbol myristate acetate (PMA) (not shown).

\section{Discussion}

We have shown that stimulation of blood monocytes with $f$ MLP induces a rapid increase in cell surface expression of two adhesion proteins, Mac-1 and p150,95. The increases over basal levels of expression are very striking, in the range of 4- to 12-fold. The LFA-1 antigen on monocytes was consistently, but less strikingly increased after $f$ MLP stimulation by $1.6-$ to 2 -fold. The cell surface quantity of other monocyte surface molecules such as Mo2, HLA class I, and HLA class II was not affected by $f$ MLP stimulation, showing that $f$ MLP receptors regulate surface expression of only a subset of monocyte membrane molecules. The $f$ MLP-stimulated increase in monocyte Mac-1 and p150,95 was rapid and was not dependent on protein synthesis.

Physiołogic concentrations of the inflammatory mediators PDGF, C5a, LTB4, and TNF were also found to stimulate increased monocyte Mac-1 expression. $f$ MLP, C5a, LTB4, and PDGF have previously been found to stimulate monocyte chemotaxis, hyperadherence to endothelial cells, aggregation, and 


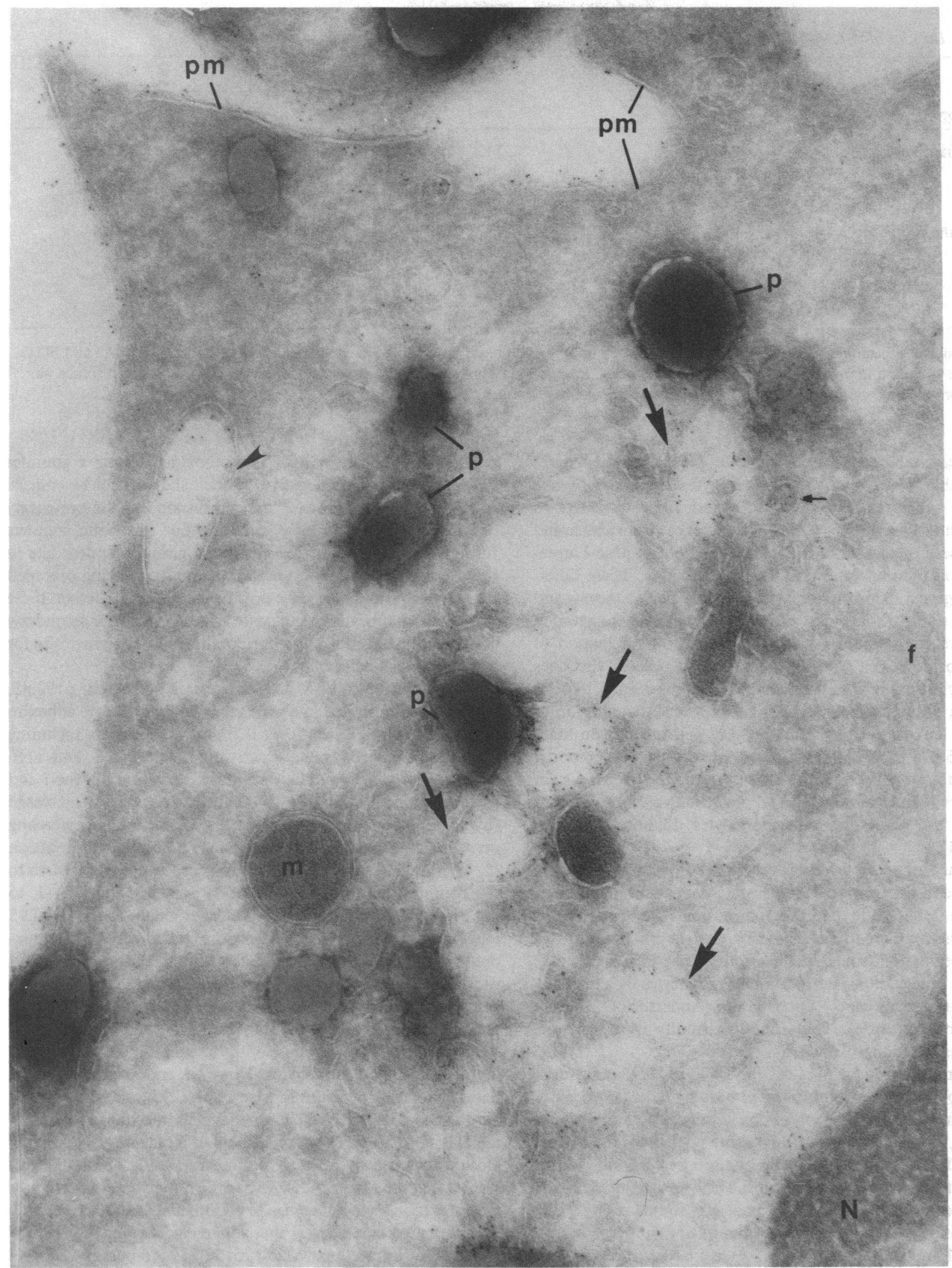

Figure 5. Frozen thin section of resting human blood monocyte. After fixation the cells were first reacted for peroxidase and then embedded in sucrose. Frozen thin sections were exposed to rabbit antiserum to p150,95 (which includes reactivities with the common beta subunit, and therefore also localizes Mac-1 and LFA-1) followed by an immunogold conjugate. Note the extensive label of immunogold along the plasma membrane ( $\mathrm{pm}$ ), as well as clear membrane bound vacuoles within the cytoplasm (arrows), and smaller more dense vesicles (small arrow). The vacuole marked with an arrowhead may well be in continuity with the plasma membrane. There is also a small amount of label on the membrane around the nucleus $(N)$. The dense reaction product for peroxidase $(P)$ is located in cytoplasmic granules. Mitochondrion $(m)$; microfilaments $(f)$. X 72,000. 
Table III. Lack of Mac-1 Mobilization on Human Alveolar Macrophages or Murine Peritoneal Macrophages*

\begin{tabular}{|c|c|c|c|c|c|}
\hline \multirow[b]{2}{*}{ Cell type } & \multirow[b]{2}{*}{ Treatment } & \multicolumn{4}{|c|}{ Specific fluorescence intensity } \\
\hline & & Mac-1 & p150,95 & LFA-1 & MHC Class I \\
\hline \multirow[t]{3}{*}{ Human alveolar $\mathbf{M} \phi$} & $4^{\circ} \mathrm{C}$ & 12 & 41 & 34 & 127 \\
\hline & $37^{\circ} \mathrm{C}, 10^{-8} \mathrm{M} f \mathrm{MLP}$ & 12 & 40 & 27 & 121 \\
\hline & $37^{\circ} \mathrm{C}, 1 \mu \mathrm{g} / \mathrm{ml}$ PMA & 9 & 29 & 29 & 97 \\
\hline \multirow[t]{4}{*}{ Murine peritoneal $\mathbf{M} \phi$} & $4^{\circ} \mathrm{C}$ & 127 & - & - & 172 \\
\hline & $37^{\circ} \mathrm{C}$ & 121 & - & - & 162 \\
\hline & $37^{\circ} \mathrm{C}, 10^{-7} \mathrm{M} f \mathrm{MLP}$ & 124 & - & - & 166 \\
\hline & $37^{\circ} \mathrm{C}, 100 \mathrm{ng} / \mathrm{ml}$ PMA & 140 & - & - & 165 \\
\hline
\end{tabular}

* Human alveolar macrophages or thioglycollate-elicited peritoneal macrophages were treated as shown for 30 min, labeled with MAb and FITC anti-Ig, and subjected to immunofluorescence flow cytometry. Results are averages of $2 / 2$ experiments. Subtracted background fluorescence was 16 to 17 for murine peritoneal macrophages, and 11 to 16 for human alveolar macrophages.

superoxide anion generation (17-20). Dose-response curves showed that compared with granulocytes, monocytes were markedly more sensitive to TNF, slightly more sensitive to PDGF, and less sensitive to $f$ MLP. Differences were also found among these mediators in the maximal extent of Mac-1 upregulation on monocytes, but not on granulocytes. These differences suggested the mediators acted directly on monocytes and granulocytes. Although in experiments with leukocyte-rich plasma we cannot rule out an intermediary participation of platelets or other cell types in the activation process, this does not detract from the physiologic relevence of our results. Platelet activation did not appear to be involved, since the addition of EDTA to leukocyte-rich plasma had little or no effect on Mac1 and p150,95 upregulation. Compared with previous reports, we have found substantially more upregulation of Mac-1 on monocytes than twofold (22), and our results are not compatible with the suggestion that monocytes do not upregulate Mac-1 (21).

Similar to a previous report on CR1 (41), we found preparative procedures can trigger temperature-dependent increases in monocyte Mac-1 and p150,95 expression. The tendency of monocytes to adhere to platelets may relate to preparation-triggered upregulation, since we have found that PDGF potently stimulates upregulation. Purification of monocytes by adherence to plastic results in Mac-1 upregulation (Anderson, D. C. Personal communication). Thus, many currently used isolation procedures functionally alter monocytes.

A latent intracellular pool of Mac-1 and p150,95 in unstimulated monocytes was demonstrated here by detergent permeabilization. Under resting conditions, significantly more Mac-1 and p150,95 were present in this latent pool than on the cell surface. The intracellular pool was quantitatively similar to the amount of Mac-1 and p150,95 on the cell surface after $f$ MLP stimulation, and no intracellular pool was found after $f$ MLP stimulation. These findings suggest that resting monocytes contain Mac-1 and p150,95 stored in intracellular vesicles, and that $f$ MLP stimulates transport of this stored Mac-1 and p150,95 to the cell surface, perhaps by vesicle fusion with the plasma membrane.

Immunogold labeling of thin sections and visualization in the electron microscope has verified that monocytes contain Mac-1 and p150,95 in intracellular vesicles that are peroxidasenegative. Blood monocytes have previously been shown to con- tain both peroxidase-positive and peroxidase-negative granules (39). The peroxidase-negative vesicles shown here to contain Mac-1 family glycoproteins may correspond to the previously defined peroxidase-negative granules, but this point requires further study. The peroxidase-negative granules appear late in maturation of promonocytes in bone marrow, being preceded by formation of peroxidase-positive granules. Whether these granules in monocytes are analogous to the specific, or secondary, granules of neutrophils, a reported intracellular storage site for Mac-1 (16), is unclear.

The importance of Mac-1, and to a lesser extent p150,95, has been demonstrated in a wide variety of granulocyte adhesion reactions (reviewed in 46). $f$ MLP and other agents including C5a, leukotriene B4, platelet activating factor, LPS, and TNF stimulate increased granulocyte surface expression of Mac-1 and p150,95 $(1,5,10,14,21,45)$. This correlates with increased (hyper-) adherence to endothelial cells, adherence and spreading on artificial substrates, and granulocyte homotypic aggregation $(5,47,48)$. These adherence functions are inhibited by MAb to the common beta subunit of Mac-1, p150,95, and LFA-1, to the Mac-1 alpha subunit, and to a lesser extent to the p150,95 alpha subunit $(8,11,13)$. Furthermore, genetically deficient patient granulocytes that lack intracellular and surface Mac-1 and p150,95 show depressed baseline adherence and a lack of $f$ MLPstimulated hyperadherence and aggregation $(5,8,9,48)$.

Less is known about the importance of these molecules on monocytes, primarily because of the greater difficulty of obtaining purified blood monocytes. However, monocytes from patients genetically deficient in Mac-1, p150,95, and LFA-1 show diminished adherence to endothelial cells and diminished chemotaxis; MAb to the common beta subunit of these glycoproteins block adherence by normal monocytes $(7,9)$. These findings suggest that the Mac-1, p150,95 and LFA-1 glycoprotein family mediates monocyte adherence to endothelial cells, and the findings presented here suggest that adherence is regulated by mobilization of intracellular Mac-1, p150,95, and possibly LFA-1.

Mac-1 has a dual function as a receptor for $\mathrm{iC} 3 \mathrm{~b}$ and as an adhesion protein $(4,13,46)$. Solubilized p150,95 binds to iC $3 b$ (49) and also may function as an adhesion protein (13). Upregulation of these molecules should enhance complement-dependent monocyte effector functions, as has been demonstrated by Yancey et al. (22). Binding of monocytes to fibronectin-coated surfaces stimulates $\mathrm{iC} 3 \mathrm{~b}$-mediated phagocytosis without affecting 
the number of Mac-1 (iC3b) receptors (50). Thus, stimulated upregulation of Mac-1, and binding of monocytes to fibronectin in the extracellular matrices underlying endothelial cells, provide two independent mechanisms for enhancing monocyte complement receptor function during extravasation.

We found that $f$ MLP-stimulated increases in the Mac- 1 and p150,95 adhesion proteins were similar in monocytes and granulocytes, although LFA-1 was increased in monocytes but not in granulocytes. Detergent permeabilization demonstrated latent, intracellular Mac-1 and p150,95 in both cell types. This suggests that adhesiveness in granulocytes and monocytes is regulated by similar mechanisms. The importance of these glycoproteins in diapedesis of both types of cells is further supported by lack of exudation of both monocytes and granulocytes in Mac-1/ p150,95/LFA-1-deficient patients $(5,51)$.

We propose that at sites of inflammation, mediators diffusing into the blood stream stimulate increased monocyte surface expression of Mac-1 and p150,95, leading to adherence to endothelial cells and localization at the inflammatory site. Upregulation in vivo may be initially less complete than at the saturating concentrations of mediators used here, and continued upregulation of Mac-1 and p150,95 during diapedesis may help mediate extravasation. Differences between monocytes and granulocytes in the dose of mediator required for Mac-1 upregulation, most notably in the 10 - to 100 -fold greater sensitivity of monocytes to TNF, may have important consequences for determining which cell type predominates in inflammatory infiltrates in vivo.

Monocytes cross the endothelium just once in their life history, and then differentiate into the diverse cells of the mononuclear phagocyte system. During differentiation, the amount of p150,95 expression increases $(25,26)$. Human alveolar macrophages express higher baseline levels of these antigens, and lack mobilizable Mac-1 and p150,95. Mouse peritoneal macrophages similarly lack mobilizable Mac-1. These findings suggest that intracellular pools of Mac-1 and p150,95 are lost during extravasation. The secretory pool of Mac-1 and p150,95 appears to be a specialized feature of circulating monocytes, of importance in crossing the endothelial barrier.

\section{Acknowledgments}

We thank W. S. Thompson for expert technical assistance and D. Anderson for discussion and contributing results on monocytes purified by adherence. We are grateful to $Y$. Jacques for the frozen-thin sectioned material.

Supported by National Institutes of Health grant CA-31799 and AM10486, Council for Tobacco Research grant 1307, Danish Medical Research grant 12-6134, and an American Cancer Society Faculty Research Award.

\section{References}

1. Harlan, J. M. 1985. Leukocyte-endothelial interactions. Blood. 65:513-525.

2. Bevilacqua, M. P., J. S. Pober, M. E. Wheeler, R. S. Cotran, and M. A. Gimbrone, Jr. 1985. Interleukin 1 acts on cultured human vascular endothelium to increase the adhesion of polymorphonuclear leukocytes, monocytes, and related leukocyte cell lines. J. Clin. Invest. 76:20032011.

3. Gallin, J. I. 1984. Neutrophil specific granules. A fuse that ignites the inflammatory response. Clin. Res. 32:320-328.

4. Springer, T. A., and D. C. Anderson. 1986. The importance of the Mac-1, LFA-1 glycoprotein family in monocyte and granulocyte adherence, chemotaxis, and migration into inflammatory sites: Insights from an experiment of nature. In Biochemistry of Macrophages (Ciba Symposium 118). Pitman, London. 102-126.

5. Anderson, D. C., F. C. Schmalstieg, M. J. Finegold, B. J. Hughes, R. Rothlein, L. J. Miller, S. Kohl, M. F. Tosi, R. L. Jacobs, T. C. Waldrop, A. S. Goldman, W. T. Shearer, and T. A. Springer. 1985. The severe and moderate phenotypes of heritable Mac-1, LFA-1 deficiency. Their quantitative definition and relation to leukocyte dysfunction and clinical features. J. Infect. Dis. 152:668-689.

6. Buchanan, M. R., C. A. Crowley, R. E. Rosin, M. A. Gimbrone, and B. M. Babior. 1982. Studies on the interaction between GP-180deficient neutrophils and vascular endothelium. Blood. 60:160-165.

7. Wallis, W. J., P. G. Beatty, H. D. Ochs, and J. M. Harlan. 1985. Human monocyte adherence to cultured vascular endothelium: Monoclonal antibody-defined mechanisms. J. Immunol. 135:2323-2330.

8. Diener, A. M., P. G. Beatty, H. D. Ochs, and J. M. Harlan. 1985. The role of neutrophil membrane glycoprotein 150 (GP-150) in neutrophil-mediated endothelial cell injury in vitro. J. Immunol. 135:537-543.

9. Buescher, E. S., T. Gaither, J. Nath, and J. I. Gallin. 1985. Abnormal adherence-related functions of neutrophils, monocytes, and Epstein-Barr virus-transformed $B$ cells in a patient with $C 3 b i$ receptor deficiency. Blood. 65:1382-1390.

10. Tonnesen, M. G., D. C. Anderson, T. A. Springer, A. Knedler, N. Avdi, and P. M. Henson. 1986. Mac-1 glycoprotein family mediates adherence of neutrophils to endothelial cells stimulated by leukotriene B4 and platelet activating factor. Fed. Proc. 45:379.

11. Beatty, P. G., J. A. Ledbetter, P. J. Martin, T. H. Price, and J. A. Hansen. 1983. Definition of a common leukocyte cell-surface antigen (Lp95-150) associated with diverse cell-mediated immune functions. $J$. Immunol. 131:2913-2918.

12. Pohlman, T. H., K. A. Stanness, P. G. Beatty, H. D. Ochs, and J. M. Harlan. 1986. An endothelial cell surface factor(s) induced in vitro by lipopolysaccharide, interleukin 1 , and tumor necrosis factor-alpha increases neutrophil adherence by a CDw18-dependent mechanism. $J$. Immunol. 136:4548-4553.

13. Anderson, D. C., L. J. Miller, F. C. Schmalstieg, R. Rothlein, and T. A. Springer. 1986. Contributions of the Mac-1 glycoprotein family to adherence-dependent granulocyte functions: Structure-function assessments employing subunit-specific monoclonal antibodies. J. Immunol. 137:15-27.

14. Springer, T. A., W. S. Thompson, L. J. Miller, F. C. Schmalstieg, and D. C. Anderson. 1984. Inherited deficiency of the Mac-1, LFA-1, p150,95 glycoprotein family and its molecular basis. J. Exp. Med. 160: 1901-1918.

15. Springer, T. A., L. J. Miller, and D. C. Anderson. 1986. p150,95, the third member of the Mac-1, LFA-1 human leukocyte adhesion glycoprotein family. J. Immunol. 136:240-245.

16. Todd, R. F. III, M. A. Arnaout, R. E. Rosin, C. A. Crowley, W. A. Peters, and B. M. Babior. 1984. Subcellular localization of the large subunit of Mol (Mol alpha; formerly gp 110), a surface glycoprotein associated with neutrophil adhesion. J. Clin. Invest. 74:1280-1290.

17. Snyderman, R., and M. C. Pike. 1984. Chemoattractant receptors on phagocytic cells. Annu. Rev. Immunol. 2:257-281.

18. Yasaka, T., L. A. Boxer, and R. L. Baehner. 1982. Monocyte aggregation and superoxide anion release in response to formyl-metionylleucyl-phenylalanine (FMLP) and platelet-activating factor (PAF). $J$. Immunol. 128:1939-1944.

19. Deuel, T. F., R. M. Senior, J. S. Huang, and G. L. Griffin. 1982. Chemotaxis of monocytes and neutrophils to platelet-derived growth factor. J. Clin. Invest. 69:1046-1049.

20. Tzeng, D. Y., T. F. Deuel, J. S. Huang, and R. L. Baehner. 1985. Platelet-derived growth factor promotes human peripheral monocyte activation. Blood. 66:179-183.

21. Arnaout, M. A., R. F. Todd III, and N. Dana. 1984. Increased surface expression of Mol on human granulocytes by chemical and phagocytic stimuli. Fed. Proc. 43:1665.

22. Yancey, K. B., J. O’Shea, T. Chused, E. Brown, T. Takahashi, 
M. M. Frank, and T. J. Lawley. 1985. Human C5a modulates monocyte Fc and C3 receptor expression. J. Immunol. 135:465-470.

23. Roberts, R. L., and J. I. Gallin. 1985. Rapid method for isolation of normal human peripheral blood eosinophils on discontinuous Percoll gradients and comparison with neutrophils. Blood. 65:433-440.

24. Antoniades, H. N., C. D. Scher, and C. D. Stiles. 1979. Purification of human platelet-derived growth factor. Proc. Natl. Acad. Sci. USA. 76: 1809-1813.

25. Miller, L. J., R. Schwarting, and T. A. Springer. 1986. Regulated expression of the Mac-1, LFA-1, p150,95 glycoprotein family during leukocyte differentiation. J. Immunol. 137:2891-2900.

26. Schwarting, R., H. Stein, and C. Y. Wang. 1985. The monoclonal antibodies anti S-HCL 1 (anti Leu 14) and anti S-HCL 3 (anti Leu M5) allow the diagnosis of hairy cell leukemia. Blood. 65:974-983.

27. Sanchez-Madrid, F., J. Nagy, E. Robbins, P. Simon, and T. A. Springer. 1983. A human leukocyte differentiation antigen family with distinct alpha subunits and a common beta subunit: the lymphocyte function-associated antigen (LFA-1), the C3bi complement receptor (OKM1/Mac-1), and the p150,95 molecule. J. Exp. Med. 158:17851803.

28. Iida, K., R. Mornaghi, and V. Nussenzweig. 1982. Complement receptor (CR1) deficiency in erythrocytes from patients with systemic lupus erythematosus. J. Exp. Med. 155:1427-1438.

29. Dimitriu-Bona, A., G. R. Burmester, S. J. Waters, and R. J. Winchester. 1983. Human mononuclear phagocyte differentiation antigens. 1. Patterns of antigenic expression on the surface of human monocytes and macrophages defined by monoclonal antibodies. J. Immunol. 130:145-152.

30. Miedema, F., P. A. T. Tetteroo, W. G. Hesselink, G. Werner, H. Spits, and C. J. M. Melief. 1984. Both Fc receptors and LFA-1 on human $\mathrm{T} \gamma$ lymphocytes are required for antibody-dependent cellular cytotoxicity (K-cell activity). Eur. J. Immunol. 14:518-523.

31. Bernstein, I. D., and S. Self. 1986. Joint report of the myeloid section of the Second International Workshop on Human Leukocyte Differentiation Antigens. In Human Myeloid and Hematopoietic Cells. E. L. Reinherz, B. F. Haynes, L. M. Nadler, and I. D. Bernstein, editors. Springer-Verlag, New York. 1-25.

32. Springer, T. A., and D. C. Anderson. 1986. Antibodies specific for the Mac-1, LFA-1, p150,95 glycoproteins or their family, or for other granulocyte proteins, in the Second International Workshop on Human Leukocyte Differentiation Antigens. In Human Myeloid and Hematopoietic Cells. E. L. Reinherz, B. F. Haynes, L. M. Nadler, and I. D. Bernstein, editors. Springer-Verlag, New York. 55-68.

33. Barnstable, C. J., W. F. Bodmer, G. Brown, G. Galfre, C. Milstein, A. F. Williams, and A. Ziegler. 1978. Production of monoclonal antibodies to group A erythrocytes, HLA and other human cell surface antigens-new tools for genetic analysis. Cell. 14:9-20.

34. Sanchez-Madrid, F., A. M. Krensky, C. F. Ware, E. Robbins, J. L. Strominger, S. J. Burakoff, and T. A. Springer. 1982. Three distinct antigens associated with human T lymphocyte-mediated cytolysis: LFA1, LFA-2, and LFA-3. Proc. Natl. Acad. Sci. USA. 79:7489-7493.

35. McMichael, A. J., N. A. Rust, J. R. Pilch, R. Sochynsky, J. Morton, D. Y. Mason, C. Ruan, G. Tobelem, and J. Caen. 1981. Monoclonal antibody to human platelet glycoprotein I. I: Immunological studies. Br. J. Haematol. 49:501-509.

36. Springer, T., G. Galfre, D. S. Secher, and C. Milstein. 1979. Mac1: a macrophage differentiation antigen identified by monoclonal antibody. Eur. J. Immunol. 9:301-306.

37. Stallcup, K. C., T. A. Springer, and M. F. Mescher. 1981. Characterization of an anti-H-2 monoclonal antibody and its use in largescale antigen purification. J. Immunol. 127:923-930.
38. Kürzinger, K., T. Reynolds, R. N. Germain, D. Davignon, E. Martz, and T. A. Springer. 1981. A novel lymphocyte function-associated antigen (LFA-1): cellular distribution, quantitative expression, and structure. J. Immunol. 127:596-602.

39. Nichols, B. A., and D. F. Bainton. 1973. Differentiation of human monocytes in bone marrow and blood: Sequential formation of two granule populations. Lab. Invest. 29:27-40.

40. Stenberg, P. E., M. A. Shuman, S. P. Levine, and D. F. Bainton. 1984. Redistribution of alpha-granules and their contents in thrombinstimulated platelets. J. Cell Biol. 98:748-760.

40a. Miller, L. J., and T. A. Springer. 1987. Biosynthesis and glycosylation of p150,95 and related leukocyte adhesion proteins. J. Immunol. 139: In press.

41. Fearon, D. T., and L. A. Collins. 1983. Increased expression of $\mathrm{C} 3 \mathrm{~b}$ receptors on polymorphonuclear leukocytes induced by chemotactic factors and by purification procedures. J. Immunol. 130:370-375.

42. Fleit, H. B., S. D. Wright, and J. C. Unkeless. 1982. Human neutrophil Fc- $\gamma$ receptor distribution and structure. Proc. Natl. Acad. Sci. USA. 79:3275-3279.

43. Todd, R. F., III, L. M. Nadler, and S. F. Schlossman. 1981. Antigens on human monocytes identified by monoclonal antibodies. $J$. Immunol. 126:1435-1442.

44. Hogg, N., L. Takacs, D. G. Palmer, Y. Selvendran, and C. Allen. 1986. The p150,95 molecule is a marker of human mononuclear phagocytes: comparison with expression of class II molecules. Eur. J. Immunol. 16:240-248.

45. Arnaout, M. A., H. Spits, C. Terhorst, J. Pitt, and R. F. Todd III. 1984. Deficiency of a leukocyte surface glycoprotein (LFA-1) in two patients with Mol deficiency. J. Clin. Invest. 74:1291-1300.

46. Anderson, D. C., and T. A. Springer. 1987. Leukocyte adhesion deficiency. An inherited defect in the Mac-1, LFA-1, and p150,95 glycoproteins. Annu. Rev. Med. 38:175-194.

47. Tonnesen, M. G., L. A. Smedly, and P. M. Henson. 1984. Neutrophil-endothelial cell interactions: Modulation of neutrophil adhesiveness induced by complement fragments $\mathrm{C5a}$ and C5a des arg and formyl-methionyl-leucyl-phenylalanine in vitro. J. Clin. Invest. 74:15811592.

48. Anderson, D. C., F. C. Schmalstieg, M. A. Arnaout, S. Kohl, M. F. Tosi, N. Dana, G. J. Buffone, B. J. Hughes, B. R. Brinkley, W. D. Dickey, J. S. Abramson, T. Springer, L. A. Boxer, J. M. Hollers, and C. W. Smith. 1984. Abnormalities of polymorphonuclear leukocyte function associated with a heritable deficiency of high molecular weight surface glycoproteins (GP138): Common relationship to diminished cell adherence. J. Clin. Invest. 74:536-551.

49. Micklem, K. J., and R. B. Sim. 1985. Isolation of complementfragment-iC3b-binding proteins by affinity chromatography. Biochem. J. 231:233-236.

50. Wright, S. D., P. A. Detmers, M. T. C. Jong, and B. C. Meyer. 1986. Interferon-gamma depresses binding of ligand by $\mathrm{C} 3 \mathrm{~b}$ and $\mathrm{C} 3 \mathrm{bi}$ receptors on cultured human monocytes, an effect reversed by fibronectin. J. Exp. Med. 163:1245-1259.

51. Bowen, T. J., H. D. Ochs, L. C. Altman, T. H. Price, D. E. Van Epps, D. L. Brautigan, R. E. Rosin, W. D. Perkins, B. M. Babior, S. J. Klebanoff, and R. J. Wedgewood. 1982. Severe recurrent bacterial infections associated with defective adherence and chemotaxis in two patients with neutrophils deficient in a cell-associated glycoprotein. J. Pediatr. 101:932-940.

52. Borregaard, N., J. M. Heiple, E. R. Simons, and R. A. Clark. 1983. Subcellular localization of the b-Cytochrome component of the human neutrophil microbicidal oxidase. Translocation during activation. J. Cell Biol. 97:52-61. 